Review

\title{
Non-Invasive Systems and Methods Patents Review Based on Electrocardiogram for Diagnosis of Cardiovascular Diseases
}

\author{
Nellyzeth Flores ${ }^{1}\left(\mathbb{D}\right.$, Marco A. Reyna ${ }^{1}$, Roberto L. Avitia ${ }^{1, * \mathbb{D}}$, Jose Antonio Cardenas-Haro ${ }^{2}$ and \\ Conrado Garcia-Gonzalez ${ }^{1}$ (D) \\ 1 Bioengineering Department, Engineering Institute, Autonomous University of Baja California, \\ Mexicali 21280, Mexico; nellyzeth.flores@uabc.edu.mx (N.F.); mreyna@uabc.edu.mx (M.A.R.); \\ cnrdgarciag@uabc.edu.mx (C.G.-G.) \\ 2 Department of Computer Science, Western Illinois University, Macomb, IL 61455, USA; \\ a-cardenas-haro@wiu.edu \\ * Correspondence: ravitia@uabc.edu.mx
}

Citation: Flores, N.; Reyna, M.A.;

Avitia, R.L.; Cardenas-Haro, J.A.;

Garcia-Gonzalez, C. Non-Invasive Systems and Methods Patents Review Based on Electrocardiogram for Diagnosis of Cardiovascular Diseases. Algorithms 2022, 15, 82. https://doi.org/10.3390/a15030082

Academic Editors: Gopi Battineni, Syed Sarosh Mahdi and

Alessandro Peretti

Received: 21 January 2022

Accepted: 21 February 2022

Published: 28 February 2022

Publisher's Note: MDPI stays neutral with regard to jurisdictional claims in published maps and institutional affiliations.

Copyright: (C) 2022 by the authors. Licensee MDPI, Basel, Switzerland. This article is an open access article distributed under the terms and conditions of the Creative Commons Attribution (CC BY) license (https:// creativecommons.org/licenses/by/ $4.0 /$ )

\begin{abstract}
Cardiovascular disease (CVD) is a global public health problem. It is a disease of multifactorial origin, and with this characteristic, having an accurate diagnosis of its incidence is a problem that health personnel face every day. That is why having all the indispensable tools to achieve optimal results is of utmost importance. Time is an essential factor when identifying heart problems, specialists look for and develop options to improve this aspect, which requires a thorough analysis of the patient, electrocardiograms being the factor standard for diagnosis and monitoring of patients. In this paper, we review patents and combined systems for the analysis of existing electrocardiogram signals, specific to cardiovascular diseases. All these methods and equipment have the purpose of giving an accurate diagnosis and a prediction of the presence of CVD in patients with positive risk factors. These are considered as the first diagnostic option, based on the guidelines already established in the field of preventive cardiology. The methodology consists of the searching of specific electrocardiography and cardiovascular disease subjects, taking as a reference the use of various patent databases. A total of 2634 patents were obtained in the consulted databases. Of that total, only 30 patents that met all the previous criteria were considered; furthermore, a second in-depth review of their information was conducted. It is expected that studying and reviewing these patents will allow us to know the variety of tools available for the different pathologies that make up CVD, not only for its immediate diagnosis because, as mentioned, the time factor is decisive for the best forecast but also to allow us to follow up on all the cases that arise, being able to provide a better quality of life to patients with CVD or even being able to lead them to a full recovery.
\end{abstract}

Keywords: cardiovascular disease; electrocardiogram; patents; diagnosis cardiovascular disease; CVD

\section{Introduction}

Cardiovascular diseases consist of a group of pathologies that in some way affect the normal functioning of the heart. Acute myocardial infarction is a complication of these, and consists of the lack of oxygenation of a part of the cardiac tissue, generating areas of necrosis, which prevents the propagation of the electrical impulse, which leads to an alteration of the rhythm and, sometimes, causes it to come to a halt completely [1]. This pathology is of multifactorial origin and can appear in the different stages of a human being, which makes its treatment difficult in a specific way. The WHO and the Pan American Health Organization have highlighted them as the main cause of premature death worldwide, considering them a red light of attention because they have not been able to reduce the number of cases, on the contrary, they have increased in recent years [2,3].

Risk factors for CVD are smoking, cholesterol, high blood pressure, diabetes mellitus, and obesity $[4,5]$. In a report published by the AHA in 2011, it is mentioned that $40.6 \%$ of cardiovascular diseases were due to high blood pressure, $11.9 \%$ to a sedentary lifestyle, 
$13.2 \%$ to a poorly balanced diet, $13.7 \%$ to smoking, and $88 \%$ to poorly controlled diabetes mellitus [6]. In these studies, it was established that it is possible to directly control blood pressure, total cholesterol, and smoking, which will lead to a significant reduction in cardiovascular risk. While for the control of obesity and diabetes mellitus, more time will be required to find formulas that serve to reduce their impact on society. A point to note is that CVD was considered a disease that affected mostly men, but over the years it has been shown that this is not entirely true, since there are underlying pathologies, such as diabetes and smoking, that cause more CVD problems in women $[7,8]$.

The electrocardiogram (ECG) is the heart electrical activity record; this is considered as the "gold standard". The importance of its study and use lies in its low cost, it is a non-invasive method and it provides information about the patient. It even allows the identification of different cardiac pathologies based on the morphology of the signal [9-11].

There are currently two global public health problems that, although they are not part of the risk factors, have been seen to directly affect the development of CVD. One of them is environmental pollution, not only because of its impact on the environment, presenting itself as part of global warming, but as an entity that in itself causes millions of premature deaths around the world. It has been found to be associated with $60 \%$ to $80 \%$ of CVD deaths [12]. It is made up of two main composition groups: particles of different sizes and gaseous components. The particles vary in their constitution and size, depending on the emitting source; generally, they are mainly organic elements. With regard to gasses, these are more specific, since they are variations of ozone, sulfur oxides, nitrogen oxides, and carbon monoxide. These gasses react with each other and with particles in the same environment, causing the appearance of more harmful compounds [12,13]. There are studies that have observed that having a concentration of PM 2.5 at a value of $10 \mathrm{mg} / \mathrm{m}^{3}$, increases the arterial pressure of the exposed individual by $3 \mathrm{mmHg}$ in a state of rest, simply by breathing these pollutants. Therefore, when exposed to constant concentrations, the individual with normal blood pressure over time can develop arterial hypertension, which is a risk factor for the appearance of CVD [14].

A new cause of CVD that is very relevant nowadays is called COVID-19. This cause of CVD begins with respiratory symptoms, but in its natural evolution triggers a generalized systemic inflammation in the host. Although initially identified as a totally respiratory disease, the inflammation affects countless organs and systems, producing a series of damages and sequelae with which surviving patients will have to live [15]. In a case series of 146 COVID-19-positive hospitalized patients in China, it was reported that $20 \%$ had a cardiac injury and a $51.2 \%$ higher mortality compared to $4.5 \%$ of patients with cardiac injury versus those without it [16].

Risk factors are a guide to determine if a person is at an increased risk of developing a disease, but they are not enough to provide a diagnosis, since a diagnostic method is needed to be certain of the presence of a CVD. Taking into consideration the two previously mentioned public health problems, an increase in CVD cases is expected in the coming years related to their sequelae, in addition to the fact that the current approach to these pathologies must be updated and both situations considered, either due to exposure to certain pollutants and having suffered from COVID-19 disease, as sufficient reasons to request heart studies, specifically, requesting an electrocardiogram. On the contrary, we have that the culture of preventive medicine is not a priority in many countries, so conducting control studies is not the rule. Another point against it is the patients' affordability to the costs of medical services, so if they are asked to take a study without being ill, they would prefer not to do it. The importance of having diagnostic equipment that is not invasive, and that does not require a lot of time for its preparation, as well as having accessible costs, will allow the patients' willingness to consider preventive medicine as a way of taking care of their health. That is why it is necessary to know emerging electrocardiography equipment options that meet those needs. Nowadays it could be the difference between death or survival. 


\section{Materials and Methods}

To carry out this patent review, the methodology consisted of a specific search on the subject of electrocardiography and cardiovascular diseases, taking as reference the use of various patent databases for its broader search field. The search engines used included the following: SCOPUS [17], Google Patents [18], SIGA [19], WIPO (Switzerland) [20], Espacenet [21] and USPTO [22].

\subsection{Keywords}

The main keywords used were: pollution, electrocardiography, cardiovascular disease. The related words that were used are: ECG, EKG, electrocardiogram, heart attack, CVD, and ischemia. The AND and OR connectors were used to create search strings that would better delimit the topic.

\subsection{Inclusion and Exclusion Criteria}

In order to determine the functional patents that provide the necessary and adequate information for the diagnosis of cardiovascular diseases, the inclusion and exclusion criteria were established, as described in Table 1. These criteria comprise most of the factors of risk for CVD, and the ECG, which is the non-invasive diagnostic standard to determine the presence or probability of developing any of those [23].

Table 1. Inclusion and exclusion criteria.

\begin{tabular}{llll}
\hline & \multicolumn{1}{c}{ Inclusion: } & \multicolumn{2}{c}{ Exclusion } \\
\hline 1. & 2010 onwards. & 1. & 2009 towards. \\
2. & ECG. & 2. & Abandoned. \\
3. & Risk Factors. & 3. & Not English/Spanish version. \\
4. & Actives. & 4. & Not ECG. \\
5. & Methods. & 5. & Not risk factors. \\
6. & Systems & 6. & Genetics. \\
7. & Medical simulation. & 7. & Blood studies \\
8. & CVD & & \\
9. & Modeling. & & \\
\hline
\end{tabular}

\subsection{Classification}

For a better search of electrocardiography patents, the classification provided by WIPO (World Intellectual Property Organization) [24] was identified. That it is standardized for all countries, and with that classification number you can determine the area and subareas that best describe the invention, as well as its application area. In this case, the G16H50/30 classification was used to search for patents (Figure 1).

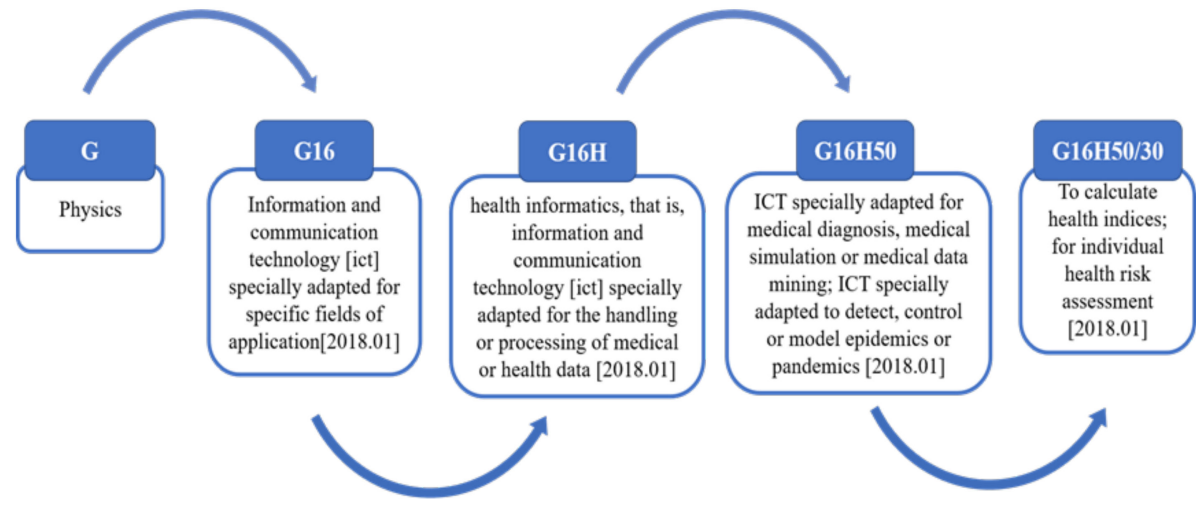

Figure 1. WIPO classification for patents in the area of health and informatics. 


\section{Results}

A total of 2634 patents were obtained from the six databases consulted. The analysis and revision of the patents were carried out with scrutiny with the application of what was obtained with the inclusion and exclusion criteria. First, the exclusion criteria were used, eliminating the patents that were abandoned, published before 2010, with a language other than Spanish or English, that did not use the ECG, and that were invasive for the patients, that is, that they used genetic and blood studies. This later made it possible to review the inclusion criteria of the remaining patents, of which 100 were obtained that superficially complied with both.

The second revision of the patents was more exhaustive. At this point, we sought to be more objective and to make greater scrutiny of the information obtained from the patents. At this point, 70 patents were discarded due to a lack of information. This was re-analyzed using the inclusion and exclusion criteria already mentioned in case any point had been overlooked. The reasons that were considered for exclusion in the second stage were the amount of information provided in the patent documentation. This refers to the fact that many of those patents only described the equipment in a physical way or the elements of the methods; no relevant data for the operation were included and there was no further information regarding the processes and methodologies themselves, they only consisted of the diagram and a brief description. A total of 30 patents were obtained that met the necessary points for analysis and the points that were considered for research (Figure 2).

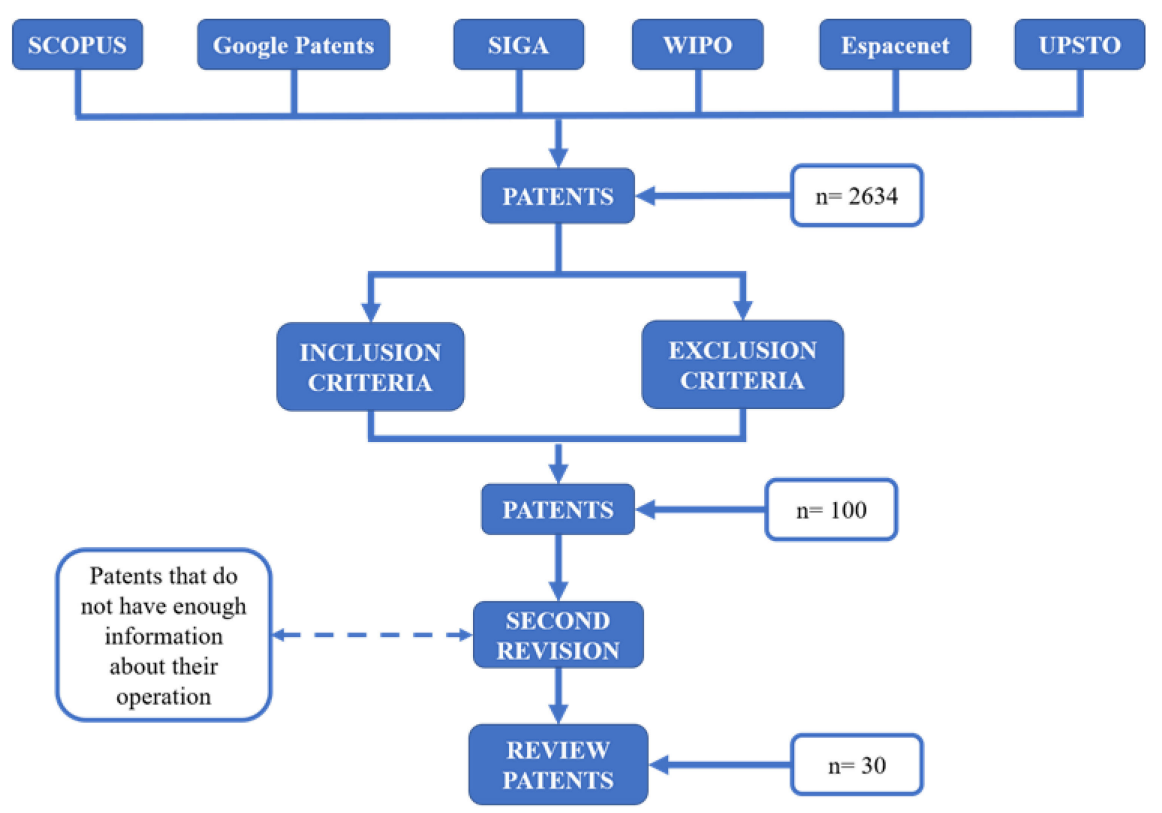

Figure 2. Patent analysis flow chart.

The technical data of the 30 patents are specified as follows: patent number, year of publication, developers or inventors, country of origin, and in which database they can be found if available. Due to the characteristics and procedures that are required for the registration of any patent, not all inventions have an international patent number. The quantity of the patents by country is described in Figure 3. Most of the patents come from the USA. This is due to the limitation of the language that was put in the inclusion criteria, although it mainly influences the fact that the industry in the field of medical equipment is carried out in that country, both by the companies and in the area of research from universities. Another point to emphasize is that most of the patents are developed by the same companies as advances in their already recognized models, which means constant improvements and updates in relation to the advances that are made for different types of diseases. 


\section{PATENTS BY COUNTRY}

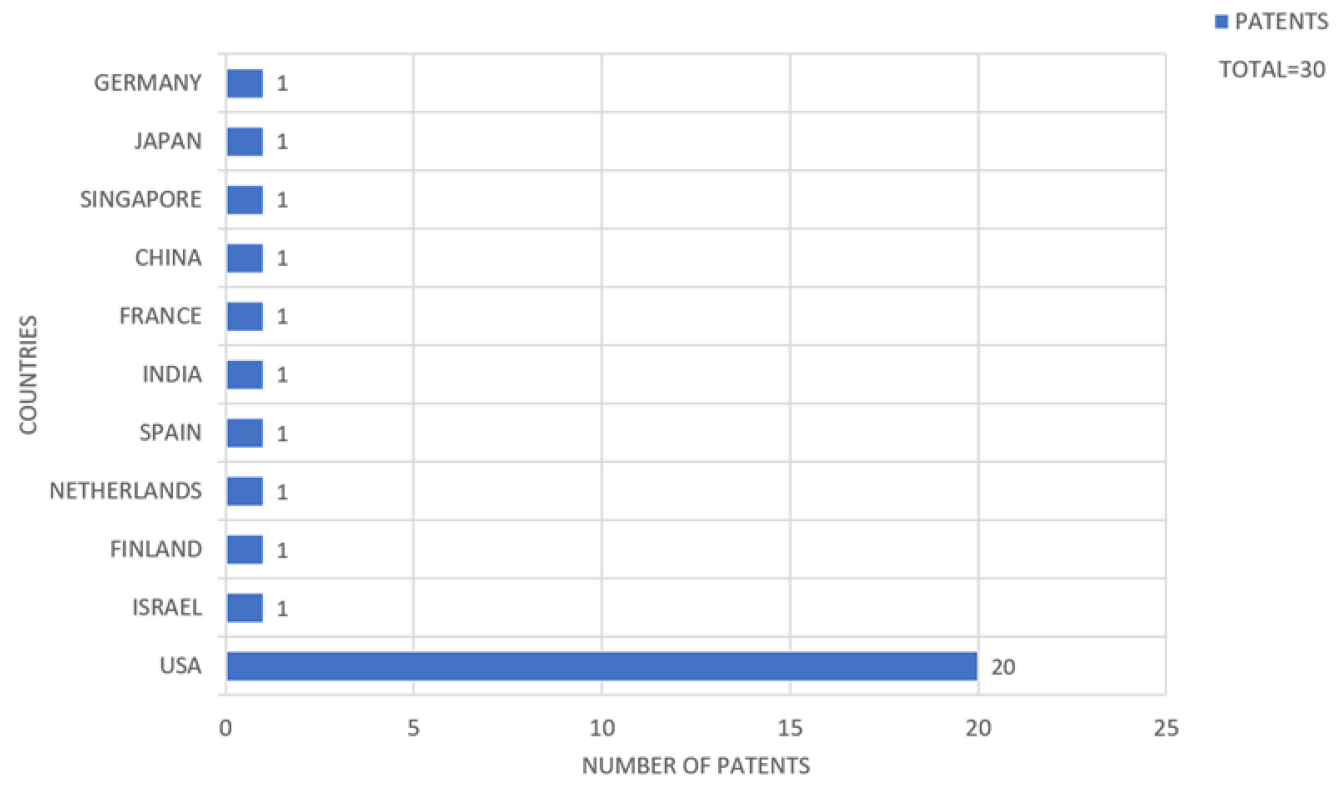

Figure 3. Number of patents per country.

\subsection{Machine and Process for the Automatic Localization of Sources of Biological Rhythm Disorders}

(WO 2010/042826 A1) USA, 2010. This consists of a method, system, and apparatus that has the capacity to detect, diagnose and treat biological rhythm disorders. It allows the analysis to be carried out in real-time, managing to detect disorders that by conventional means can be confused or not located. It identifies localized sources of atrial fibrillation. The advantage presented by this patent is that, by identifying the cause of the arrhythmia, it helps with the treatment so that the appropriate one is used and eliminates the potential disorder using minimally invasive techniques [25].

\subsection{Method and System for Cardiovascular System Diagnosis}

(US 7771364) Israel, 2010. The method consists of measuring the patient's pulse wave signals during rapid excitation of the cardiovascular system. These signals are analyzed and the indicators that reflect the response of that excitation are calculated. Cardiovascular arousal occurs by controlled breathing, which maintains a predefined frequency of approximately $0.1 \mathrm{~Hz}$. Using this frequency generates a response in the HRV, which has proven to be a predictor of sudden death. Furthermore, it is shown that presenting a failure in the parasympathetic system is highly correlated with the risk of presenting coronary events [26].

\subsection{Method and Software for Cardiovascular Assessment and Risk Detection}

(US 2011/0208434) USA, 2011. This patent presents an improvement to the method for evaluating a person's risk of developing CVD. This is performed through the analysis of multiple factors, including the Framingham Criteria, in addition to adding chronic inflammation, insulin resistance, and kidney dysfunction. Once the data are collected, each factor is quantified using the categorized risk levels for each test performed, in addition to analyzing the combinations of specific risks that increase the probabilities of CVD, such as chronic inflammation with insulin resistance [27].

\subsection{System and Method for Separating Cardiac Signals}

(US/7941205) USA, 2011. This patent consists of obtaining the ECG signal through sensors. This signal will be analyzed by a computer module, which uses independent components to separate the different recorded signals. Those signals can then be reanalyzed by doctors and thus identify a heart condition. The advantage it presents is that, 
by individually analyzing the signals, it can detect or predict an abnormal condition in the patient's heart, which is observed when a warning notice is generated [28].

\subsection{System and Method for Mapping Complex Fractionated Electrocardiogram Information}

(US/8229545) USA, 2012. This system takes the information provided by an electrode, which receives the information from the electrocardiogram and measures its location. Memory can also be provided in which to store the information. Data can be analyzed using both time domain and frequency domain information to create a three-dimensional model. By representing the mapping of the heart along with the measurements obtained, it allows for finding the approximate point where the cardiac abnormality is happening [29].

\subsection{ECG Data Monitor}

(US/2012/0311092) Finland, 2012. This patent is a system that provides a simple and low-cost ECG. It consists of monitoring the ECG signal connected to a server or cloud, in which the access is achieved through a mobile network connection where a cell phone works as a gateway. This allows the collection of signal data during a previously established period of time [30].

\subsection{System for Managing Cardiovascular Health Status}

(EP 2544111A1) The Netherlands, 2013. This is a system that allows the calculation of scores to determine the risk factor of a patient, in addition to giving a recommendation for treatment and/or change in lifestyle. It includes an input device, an interpreter of risk factors, as well as a calculator, in order to give a value that indicates that risk, as well as observations for lifestyle changes and possible treatments [31].

\subsection{Electrocardiographic Assessment of Arrhythmia Risk}

(US/8437839) USA, 2013. This consists of a system and method that indicate a risk of arrhythmia in a patient using a graphic analysis of the ECG. In order to estimate the risk of arrhythmia, you must first obtain the patient's electrocardiographic signals. Then, it averages the different signals and leads, and the amplitude and duration of each. In addition, it determines the significance of each deflection based on whether the amplitude of that deflection exceeds a threshold and estimates the risk of arrhythmia in the patient based on at least one of a number, the amplitudes, and the duration of the significant deviations [32].

\subsection{Programmable-ECG Sensor Patch}

(US/8688189). USES, 2014. This is a programmable-ECG sensor for the detection of risk patterns based on programmed criteria. It is a non-invasive method. Programming allows one or more control parameters to be selected and alarms for any data out of range are indicated at the same time. The programmable patch works in conjunction with an external programming unit to select detection monitoring parameters. The sensors can be programmed to detect a specific ECG pattern that is characteristic of a pathology. It can even be programmed in response to a cardioactive drug being evaluated. This serves to identify pathologies and see the response to treatment [33].

\subsection{Cardiac Performance Monitoring System for Use with Mobile Communication Devices}

(US/8700137) USA, 2014. This is a device and method to monitor the heart health of a patient, using a computer or smartphone that contains an accelerometer. Through the placement of electrodes on the patient, it allows the measurement of the electrical signals and vibrations in the chest caused by the heartbeat. With this information, an earthquake cardiogram (SCG) and an electrocardiogram can be generated. These measures can provide an early warning of possible heart problems and signal the need for the patient to seek treatment before a fatal cardiac event [34]. 


\subsection{Method and Device for Early Detection of Heart Attack}

(US/9161705) USA, 2015. This is a device and method for the characterization of cardiac performance and the detection of anomalies. It analyzes and characterizes cardiac electrophysiological signals that aid in the diagnosis of myocardial ischemia before a heart attack. This is accomplished by acquiring the electrophysiological signal, and in it, identifying changes in the slope of the ST segment, the T wave, and correlating the change between the ST segment and the T wave [35].

\subsection{System, Computer-Implemented Method and Computer Program Product for Individualized Multiple-Disease Quantitative Risk Assessment}

(EP 3096253A1) (US2016/0342764) Spain, 2015. This is a system for multiple diseases. It contains: a database that stores the clinical data of the patient and its registry variables (eg ECG); an analysis module to process the physiological signals and calculate the prognostic parameters; a risk assessment module to estimate risk factors, and a quantitative assessment of the risk of multiple diseases, which takes into account the information previously obtained to indicate the result of each of the events and conditions to be evaluated that have occurred [36].

\subsection{Method and Device for Assessing the Risk of Cardiovascular Complications}

(US 2015/0297098) USES, 2015. This uses optical capillaroscopy of the nail fold of a finger in a state of reactive hyperemia. The pulse wave velocity and the level of upper limb endothelial function are measured simultaneously in relation to the $\mathrm{R}$ peak on an electrocardiogram and a $\mathrm{K}$ index for the risk of cardiovascular complications [37].

\subsection{QRS Complex Identification in Electrocardiogram Signals}

(US/9414761) India, 2016. This is a method that consists of receiving and filtering the ECG signal passing through a low pass filter and a high pass filter. The filtered signal is processed to identify the maximum amplitude peak of R of the QRS complex. Subsequently, the $\mathrm{Q}$ and $\mathrm{S}$ peaks of the same complex are identified [38].

\subsection{Method and Device to Predict Adverse Cardiovascular Events and Mortality from an Electrocardiogram-Based Validated Risk Score}

(US2016/0256064) USA, 2016. This is a method to predict the risk of presenting a cardiovascular event and the mortality that the patient presents by the analysis of risk factors. The analysis is carried out as follows: record the patient's ECG; analyze the obtained ECG for the presence of wave disturbances; calculate the risk score based on the findings of the ECG analysis, and predict the risk of the presence of possible cardiovascular events based on the score of the findings. In practice, it is mentioned that this risk analysis allows the doctor to offer suggestions to their patients on measures to take to reduce the risk of suffering a CVD [39].

\subsection{Systems and Methods for Monitoring and Controlling a Cardiovascular State of a Subject}

(WO 2016/022989) USA, 2016. This is a system and method to monitor and control the cardiovascular system of a patient. It is carried out by acquiring the data of the heart rate and blood pressure, determining the trajectory time and whether or not they exceed the preset thresholds. It also allows us to observe the effect that some vasopressor treatments may have, monitoring the data before and after the intake of these drugs [40].

\subsection{System and Methods for Cardiovascular Blood Flow and Musculoskeletal Modeling for Predicting Device Failure or Clinical Events}

(WO 2016/168017) USA, 2016. This is a system and method implemented in the computer to evaluate the effect of the patient's musculoskeletal activities and their disease. It consists of receiving vascular and musculoskeletal models of the patient at rest and in activity. With these models, an analysis of fluid dynamics and a structural mechanical 
simulation are carried out. In this way, the risk of a disease or a clinical event is estimated based on fluid dynamics and mechanical performance [41].

\subsection{Automatic Method to Delineate or Categorize an Electrocardiogram}

(US 2017/0112401) France, 2017. This is a method that allows the delimit and labeling of an ECG signal using a neural network (NN). The NN was trained using around $85,000 \mathrm{vECGs}$ and was evaluated on a dataset that includes 20,000 patients who were not used in the training phase [42].

\subsection{Printed ECG Electrode and Method}

(US 2017/0332928) USA, 2017. This consists of a dry and flexible electrode, which is composed of multiple layers of carbon nanotubules (MWCNT) and polydimethylsiloxane (PDMS). This type of electrode can be used to monitor physiological signals, mainly those of the ECG. The electrode was better in terms of identifying the typical characteristic components of the ECG: P wave, QRS complex, and T wave [43].

\subsection{Analysis of Electrocardiogram Signals}

(US/8,934,964 B2) USA, 2015. This method consists of a graphical representation of a complete ECG cardiac impulse, which presents the lines of the basic isoelectric variables. The process begins with obtaining the signal, digitizing it, identifying the level of the PT interval, finding the highest point of the $\mathrm{R}$ wave, marking the reference points, and aligning the complexes using those reference points temporarily. This is achieved by obtaining a baseline for that plot and involves the non-uniform sampling of the complex [44].

\subsection{Cardiovascular Assist System That Quantifies Heart Function and Facilitates Heart Recovery}

(WO 2018/053504) USA, 2018. This consists of a device and method that use a heart pump to obtain the values of the patient's cardiovascular function. The system quantifies the functioning of the heart by taking certain parameters and signals (motor and blood pressure), then carries out the calculations to measure cardiovascular function. Among the measurements obtained are left ventricular pressure and contractility. This allows for evaluating the recovery of the patient and also if any pathology occurs [45].

\subsection{Monitor Recorder-Implemented Method for Electrocardiography Value Encoding and Compression}

(US/9730641B2) USA, 2017. This consists of a method of collecting, coding, and compression of electrocardiographic values. The ECG data obtained during monitoring consist of a two-step compression algorithm executed by the monitors. This type of compression is higher than other Holter-type monitors. The advantage this offers is that of facilitating long-term ECG tracing, which favors the diagnosis of different arrhythmias. Furthermore, the placement of the required patches in the human anatomy for the collection of the data is not invasive and does not cause any discomfort. All these represent a good option for both patients [46].

\subsection{System and Method for Assessing Clinical Event Risk Based on Heart Rate Complexity}

(WO/2019/160504) Singapore, 2019. This consists of a method to assess clinical risks for CVD based on the complexity of the individual's heart rate. The method consists of receiving the information from the device along with the measurement of the patient's physiological data. A heart rate time series is then generated from the data obtained. This then computes a set of entropy data from the heart rate time series; calculates the HRC index from the entropy data; compares the HRC index with predefined reference data, and generates a hazard signal in response to a determination from said comparison. The HRC index is indicative of higher risks of clinical events for the patient [47]. 
3.24. Continuous Detection and Monitoring of Heart Arrhythmia Using Both Wearable Sensors and Cloud-Resident Analyses

(WO 2019/108426) USES, 2019. This consists of a system and method that allow the patient to be continuously monitored in order to determine when a cardiovascular event is likely to occur. This is carried out by the interaction of various sensors placed on the patient. With the records obtained, they are analyzed with a classifier that is already programmed, which can be accessed through the cloud or a remote system. These types of classifiers reduce false positives [48].

\subsection{Electrocardiograph with Extended Lead Function and Extended Lead Electrocardiogram Deriving Method}

(US/8359090B2) Japan, 2013. This method consists of an extended ECG lead function. Using a potential detector, it determines the values of the 12 leads of the ECG, storing the data in memory, and through an arithmetic operation using the alpha coefficients (which represents a relationship between the different leads), the reconstruction of the extended signal in a waveform is observed on the display monitor. The theory used for the 12-lead relationship was based on the dipolar model of the heart, in which a potential V of an arbitrary lead can be determined by an array of the potentials [49].

\subsection{ECG Analysis for Diagnosis of Heart Failure and Cardiovascular Disease Using Signals Obtained from an Implantable Monitor}

(US 2019/0209037) Germany, 2019. This consists of an implantable device to monitor the patient's electrocardiogram. It has a detection element to determine the values and indicate abnormalities of the electrocardiographic signal of the heart. The device processor is configured prior to placement. It handles the parameters of the $\mathrm{R}$ and $\mathrm{S}$ wave relationship of the QRS complex [50].

\subsection{Multi-Channel Real-Time Cardiovascular Performance Evaluation System and Method}

(WO 2020/086112) China, 2020. This consists of a real-time cardiac performance evaluation system. It is made up of a multichannel device, a unit for measuring ECG signals, and another for reconstructing those signals. The algorithm used marks the ECG signals to be able to make a comparison with the original signal and extract the peak values of the complete ECG signal, not just one wave. With this, the patient can be evaluated and determined if they have any cardiovascular disease and the position of the lesions that can be found in that heart disease [51].

\subsection{Systems, Devices, and Methods for Non-Invasive Cardiac Monitoring}

(WO2020/205987) USA, 2020. This is a non-invasive system, method, and device for cardiovascular diseases. Its principle is the monitoring of a cardiac parameter, such as blood pressure and ECG. You can also get dynamic change logs through accelerometers. It receives the information of the patient's cardiac data, processes it, and analyzes it using the previous programming of the parametric values, showing whether or not these are in accordance with the pre-established values [52].

\subsection{Systems and Methods for Predicting Atrial Arrhythmia}

(WO 2020/190922) USA, 2020. This is a device and system which includes an arrhythmia predictor configured to receive information on the patient's physiological performance, and determine the risk that an atrial tachyarrhythmia may present. It also allows, if a high risk is identified, an alarm to be activated and for more careful monitoring of the patient to be maintained before any event occurs [53].

\subsection{Compact Mobile Three-Lead Cardiac Monitoring Device}

(WO 2020/232040) USA, 2020. This is a three-lead cardiac monitoring device. It is a mobile system that allows for the detection and diagnosis of cardiac events. The apparatus 
includes two integrated hand electrodes and two chest electrodes arranged on two pivoting arms that are capable of retracting into the compartments, allowing for a compact size when the device is not in use [54].

\section{Discussion}

In the analysis carried out during the search, a large number of patents with interesting operating principles were detected, but which for unknown reasons have been abandoned, such as, for example, the US patent 2017/0065194A1 "Cardiovascular Detection System And Method" [55], which establishes an analysis of the ECG to determine the presence of cardiovascular risk, and the US patent US 2014/0194758 A1 "System and method for evaluating an electrophysiological signal" [56], which performs a reconstruction of the electrocardiographic signal derived from a non-transitory model, which through the team manages to identify the pathological event and carry out a reconstruction of the event. It was also observed that the majority of patents correspond to companies that make improvements to existing models. In the case of patents developed by universities and their research departments, although their designs are present, they are not available in the market, and the information available in this regard is only that provided in the patent documents. The causes of this lack of information flow and follow-up to the product of the accepted and current patents are unknown.

The patents obtained can be classified into four groups, depending on their operation. The first group corresponds to the analysis and reconstruction of ECG signals. The patents that make up this group are the following: 3.4, 3.5, 3.6, 3.8, 3.10, 3.14, 3.18, 3.22, 3.25, 3.27, and 3.30 .

Analyzing and reconstructing the signals facilitates their study, and with the information collected, patient data can be created through a simulation. This avoids the need to send the patients for medical analysis again. In this way, various behavioral scenarios are proposed, either, generating future cases if the circumstances that led to that failure are followed, or the implementation of treatment possibilities, such as surgery, medications and pacemaker.

The second group corresponds to the patents that function as support for the treatments, which we find are: 3.1, 3.9, 3.16, and 3.21. This group of patents is the smallest in quantity, in its favor, in addition to providing an analysis of the patient's condition, it allows treatments to be used depending on the type of CVD that the patient presents. This supports a higher success rate in addition to subsequent revisions.

The third group is of the patents that identify abnormal rhythm patterns, that is, identify arrhythmias, based on the normal values of the ECG tracing. These patents are: $3.1,3.2,3.4,3.5,3.8,3.9,3.10,3.11,3.13,3.15,3.16,3.19,3.20,3.21,3.24,3.26,3.29$, and 3.30.

This is the largest group in the number of patents, and the advantage they present in the diagnosis is that they provide a more specific analysis to this group of pathologies, which are considered silent since their existence is not known until severe symptoms appear in the patient. Being able to identify abnormal rhythm patterns in the early stages guarantees recovery in the shortest time, in addition to being a tool that is easy to acquire and use.

The fourth group consists of the patents: $3.3,3.7,3.12,3.15,3.17,3.21,3.23$, and 3.28. These, in addition to taking into account ECG data, are supported by accelerometers, and also consider risk factors, as well as other physiological measurements, such as heart rate, blood pressure, and fluid dynamics. This type of measurement gives a more general overview of the patient's condition since it allows for evaluating the consequences that may occur in the rest of the body caused by CVD.

Although the patents were divided into four groups based on the function they present, there were some that were part of two or more groups, due to the variety they present in their functions. This reflects the same variability that CVDs present, which must be seen from different points of view to make a more complete analysis, not only to diagnose but also to include treatment and follow-up. 
Of these, five patents can be highlighted for the combination of elements that characterize their operation. The patent 3.1 (WO 2010/042826 A1), which, as mentioned, through the electrodes and the system detects anomalies for diagnosis, can also be used for the treatment of patients with pacemakers since it allows the use of potential to correct rhythm disorders. Patent 3.9 (US/8688189), due to its patch modality, makes it more comfortable for the patient, which makes it possible to detect abnormal rhythm patterns, either due to disease or medication. It also allows for the monitoring of the patient's response to treatments and evaluating their effectiveness. Patent 3.11 (US/9161705) focuses mainly on detecting regions of cardiac ischemia; it is programmed to identify specific patterns of the ST segment and the T wave, comparing them with normal values, which are characteristic areas where cardiac muscle tissue damage can be seen. Finally, patent 3.27 (WO 2020/086112), where the main feature is the real-time analysis since it not only focuses on ECG measurements but also saves the information to be able to make reconstructions of those signals after having been registered. Unlike other equipment that only identifies by wave, in this case, it completely evaluates the complete signal complex, which makes it possible to obtain a more accurate position of the lesion caused by CVD.

These patents raise what could be a breakthrough as part of preventive medicine. Being able to analyze, not only through the interrogation and clinical history of patients but also having first-hand physiological data from the patient, can lead to a reduction in CVD cases with irreversible results. As was established from the beginning, CVD is a public health problem with a growing number of cases each year and with a high mortality rate, which means it favors treatment in the earlier stages of CVD that is present in individuals.

\section{Conclusions}

This review has documented a variety of patents related to the diagnosis of cardiovascular diseases that can have an impact on the research area with the combination of several protocols in the biomedical area. As it has been established, CVD is a global public health problem that has been increasing over recent years. It is a problem that no matter how much it is has been sought to be eradicated, this has not been possible yet because it is of multifactorial origin, and because of this same diversity, there is no single diagnostic method that is effective for all cases. It takes the combination of different studies, such as ECGs, laboratory, and a patient's clinical history to be able to give a presumptive diagnosis, but this occurs only when the patient already has important symptoms and whose stage of evolution is already too late to implement first-line treatments.

Each disease included in this group has its own characteristics and there are some that have a less encouraging prognosis than others, so early detection is what improves the patient's evolution to an optimal state of health.

Preventive medicine is the best ally in these cases, so having more specific tools for all the pathologies that are part of CVD and suggesting their use in patients with positive risk factors can help predict the chances that a person develops any CVD. In addition to allowing its detection in early stages, it makes treatments more effective. That is why the review of relatively new technologies in the field of ECG study and complementary methods allows us to have the appropriate tools with which to begin to observe a decrease in the cases of this pathology, in addition to having a better prognosis for those patients who are already dealing with CVD. Furthermore, taking into account that due to the fact that they have presented new causes to develop a CVD, complementing the already established diagnostic methods and giving an opportunity to the use of these technologies should not be neglected, since this improves their long-term specificity. Another point to note is that they are intended to be non-invasive, provide comfort and safety to the patient since this guarantees the success of taking the sample to be analyzed.

Author Contributions: Conceptualization, N.F. and R.L.A.; methodology, N.F. and R.L.A.; formal analysis, M.A.R.; software, J.A.C.-H. and C.G.-G.; writing-review and editing, J.A.C.-H. and C.G.-G. All authors have read and agreed to the published version of the manuscript. 
Funding: This research received no external funding.

Institutional Review Board Statement: Not applicable.

Informed Consent Statement: Not applicable.

Data Availability Statement: Not applicable.

Acknowledgments: We want to express our gratitude to the National Council of Science and Technology of Mexico for its invaluable scholarship to support these studies.

Conflicts of Interest: The authors declare no conflict of interest.

\section{References}

1. Jameson, J.L.; Fauci, A.S.; Kasper, D.L.; Hauser, S.L.; Longo, D.L.; Loscalzo, J. Harrison's Principles of Internal Medicine, 20th ed.; McGraw-Hill: Guaynabo, Spain, 2018.

2. World Health Organization. 2018. Available online: http:/ /www.who.int/en/ (accessed on 20 January 2022).

3. WHO. World Health Statistics Monitoring Health for the SDGs, Sustainable Development Goals. 2020. Available online: https:/ / apps.who.int/iris/handle/10665/332070 (accessed on 20 January 2022).

4. $\quad$ Bays, H.E.; Taub, P.R.; Epstein, E.; Michos, E.D.; Ferraro, R.A.; Bailey, A.L.; Kelli, H.M.; Ferdinand, K.C.; Echols, M.R.; Weintraub, H.; et al. Ten things to know about ten cardiovascular disease risk factors. Am. J. Prev. Cardiol. 2021, 5, 100149. [CrossRef] [PubMed]

5. $\quad$ D’Agostino Sr, R.B.; Vasan, R.S.; Pencina, M.J.; Wolf, P.A.; Cobain, M.; Massaro, J.M.; Kannel, W.B. General cardiovascular risk profile for use in primary care: The Framingham heart study. Circulation 2008, 117, 743-753. [CrossRef] [PubMed]

6. Roger, V.L.; Go, A.S.; Lloyd-Jones, D.M.; Adams, R.J.; Berry, J.D.; Brown, T.M.; Turner, M.B. Heart disease and Stroke Statistics2011 Update A Report from the American Heart Association. Circulation 2013, 123, e18-e209.

7. Prabakaran, S.; Vitter, S.; Lundberg, G. Cardiovascular Disease in Women Update: Ischemia, Diagnostic Testing, and Menopause Hormone Therapy. Endocr. Pract. 2022, 28, 199-203. [CrossRef] [PubMed]

8. Humphries, K.H.; Izadnegadar, M.; Sedlak, T.; Saw, J.; Johnston, N.; Schenck-Gustafsson, K.; Merz, C.B. Sex differences in cardiovascular disease-Impact on care and outcomes. Front. Neuroendocrinol. 2017, 46, 46-70. [CrossRef] [PubMed]

9. Nayak, S.K.; Banerjee, I.; Pal, K. 24-Electrocardiogram signal processing-based diagnostics: Applications of wavelet transform. In Woodhead Publishing Series in Electronic and Optical Materials, Bioelectronics and Medical Devices; Pal, K., Kraatz, H., Khasnobish, A., Bag, S., Banerjee, I., Kuruganti, U., Eds.; Woodhead Publishing: Cambridge, UK, 2019; pp. 591-614. ISBN 9780081024201. [CrossRef]

10. Xiong, P.; Xue, Y.; Zhang, J.; Liu, M.; Du, H.; Zhang, H.; Hou, Z.; Wang, H.; Liu, X. Localization of myocardial infarction with multi-lead ECG based on DenseNet. Comput. Methods Programs Biomed. 2021, 203, 106024. [CrossRef] [PubMed]

11. Liu, X.; Wang, H.; Li, Z.; Qin, L. Deep learning in ECG diagnosis: A review. Knowl.-Based Syst. 2021, 227, 107187. [CrossRef]

12. Rajagopalan, S.; Al-Kindi, S.G.; Brook, R.D. Air Pollution and Cardiovascular Disease: JACC State-of-the-Art Review. J. Am. Coll. Cardiol. 2018, 72, 2054-2070. [CrossRef] [PubMed]

13. Bourdrel, T.; Bind, M.-A.; Béjot, Y.; Morel, O.; Argacha, J.-F. Cardiovascular effects of air pollution. Arch. Cardiovasc. Dis. 2017, 110, 634-642. [CrossRef]

14. Watkins, A.; Danilewitz, M.; Kusha, M.; Massé, S.; Urch, B.; Quadros, K.; Nanthakumar, K. Air Pollution and Arrhythmic Risk: The Smog Is Yet to Clear. Can. J. Cardiol. 2013, 29, 734-741. [CrossRef] [PubMed]

15. Balachandar, V.; Mahalaxmi, I.; Subramaniam, M.; Kaavya, J.; Kumar, N.S.; Laldinmawii, G.; Cho, S.G. Follow-up studies in COVID-19 recovered patients-Is it mandatory? Sci. Total Environ. 2020, 729, 10. [CrossRef] [PubMed]

16. Mahfouz, R.A.; Arab, M.; Abdelhamid, M.; Elzayat, A. Fragmented QRS complex is an independent predictor of plaque burden in patients at intermediate risk of coronary artery disease. Indian Heart J. 2019, 71, 394-399. [CrossRef] [PubMed]

17. Elsevier. SCOPUS. 2019. Available online: https://www.scopus.com/freelookup/form/author.uri (accessed on 20 January 2022)

18. Google. Google Patents. 2019. Available online: https://www.google.com/?tbm=pts (accessed on 20 January 2022).

19. IMPI. SIGA. 2019. Available online: https://siga.impi.gob.mx/newSIGA/content/common/principal.jsf (accessed on 20 January 2022).

20. WIPO. Divulgacion de Information. 2003. Available online: http://www.wipo.int/sme/es/documents/disclosing_inf.htm (accessed on 20 January 2022).

21. OEP. ESPACENET. 2019. Available online: https://lp.espacenet.com/ (accessed on 20 January 2022).

22. United States Government. USPTO. 2019. Available online: https: / /www.uspto.gov/ (accessed on 20 January 2022).

23. WHO. WHO List of Priority Medical Devices for Management of Cardiovascular Diseases and Diabetes. 2021. Available online: https:/ /apps.who.int/iris/bitstream/handle/10665/341967/9789240027978-eng.pdf (accessed on 20 January 2022).

24. WHO. WIPO. 2019. Available online: https:/ /www.wipo.int/portal/en/index.html (accessed on 20 January 2022).

25. Narayan, W.-J.; Rappel, S. Machine and Process for The Automatic Localization of Sources of Biological Rhythm Disorders. 2010. Available online: https:/ / patents.google.com/patent/WO2010042826A1/en (accessed on 20 January 2022).

26. Arbel, M.O.R.; Tal, Y. Method and System for Cardiovascular System Diagnosis. 2010, Volume 2. Available online: https: / / patents.google.com/patent/US20070021673A1/en (accessed on 20 January 2022). 
27. Hersh, S.H. Method and Software for Cardiovascular Assessment and Risk Detection. 2011. Available online: https:/ / patents. google.com/patent/US20110208434A1/en (accessed on 20 January 2022).

28. Tzyy-Ping Jung, Jeng-Ren Duann, System and Method for Separating Cardiac Signals. 2011. Available online: https:/ / patents google.com/patent/US20050010120 (accessed on 20 January 2022).

29. Afonso, V. System and Method for Mapping Complex Fractionated Electrocardiogram Information. 2012. Available online: https: / / patents.google.com/patent/US8229545 (accessed on 20 January 2022).

30. Lewis, O.; William, D.; Musiol, T.; Ratingen, D.E.; Lewis, D.W.; Atlanta, G.A.; Tong Alvarez, N.M.U.; Reinartz, R.M.; Gonzalez, B.; Bhat V. ECG Data Monitor. 2012. Available online: https:/ / patents.google.com/patent/US20120311092A1 (accessed on 20 January 2022).

31. Wendy, D.J.J.D.; Waanders, L.F.; Harsh, D.; Nagaraju, B.; Marcia, A.D.; Mani, A.K. System for Managing Cardiovascular Health Status. 2013. Available online: https://patents.google.com/patent/EP2544111A1 (accessed on 20 January 2022).

32. Lux, R.L. Electrocardiographic Assessment of Arrhythmia Risk. 2013. Available online: https://patents.google.com/patent/US8 437839B2 (accessed on 20 January 2022).

33. Shennib, A. Programmable ECG Sensor Patch. 2014. Available online: https://patents.google.com/patent/US8688189B2 (accessed on 20 January 2022).

34. Albert, D.E. Cardiac Performance Monitoring System for Use with Mobile Communication Devices. 2014. Available online: https:/ / patents.google.com/patent/US8700137B2 (accessed on 20 January 2022).

35. SLakshman, B.; Tamil, S.; Nourani, M.; Gupta, G. Method and Device for Early Detection of Heart Attack. 2015. Available online: https: / / patentscope.wipo.int/search/es/detail.jsf?docId=US73626273 (accessed on 20 January 2022).

36. Domingez, R.C.H.; Bernardez, J.R.F.; Ojea, A.M.; Garcia, D.E.A. System, Computer-Implemented Method and Computer Program Product for Individualized Multiple-Disease Quantitative Risk Assessment. 2016. Available online: https://patents.google.com/ patent/US20160342764A1 (accessed on 20 January 2022).

37. Gurfinkel, Y.I.I.; Ostrozhinskij, V.A. Method and Device for Assessing the Risk of Cardiovascular Complications. 2015. Available online: https:/ / patents.google.com/patent/US20150297098A1 (accessed on 20 January 2022).

38. Bhaumik, K.J.S.B. QRS Complex Identification in Electrocardiogram Signals. 2016. Available online: https://patents.google.com/ patent/US9414761B2 (accessed on 20 January 2022).

39. Estes, N.E.H.; Durham, J.R. Method and Device to Predict Adverse Cardiovascular Events and Mortality from an Electrocardogram-Based Validated Risk Score. 2016. Available online: https:/ / patents.google.com/patent/US20160256064A1 (accessed on 20 January 2022).

40. Bighamian, R.; Hahn, J.-O.; Reisner, A.T.; Yapps, B. Systems and Methods for Monitoring and Controlling a Cardiovascular State of a Subject. 2015. Available online: https:/ / patentscope.wipo.int/search/es/detail.jsf?docId=WO2016022989 (accessed on 20 January 2022).

41. Gilwoo, G.L.C.L.; Taylor, C.A. System and Methods for Cardiovascular Blood Flow and Musculoskeletal Modeling for Predicting Device Failure or Clinical Events. 2016. Available online: https:/ / patentscope.wipo.int/search/es/detail.jsf?docId=WO2016168 017 (accessed on 20 January 2022).

42. Rapin, J.; Li, J.; Massias, M. Automatic Method to Delineate or Categorize an Electrocardiogram. 2017. Available online: https:/ / patents.google.com/patent/US20170112401A1 (accessed on 20 January 2022).

43. Atashbar, A.E.M.Z.; Chlaihawi, A.A.; Narakathu, B.B. Printed ECG Electrode and Method. 2017. Available online: https: / / patents.google.com/patent/US20170332928A1 (accessed on 20 January 2022).

44. Benjamin, S.; Shai, R.; Aaron, F. Analysis of Electrocardiogram Signals. 2015. Available online: https://patents.google.com/ patent/US8934964B2 (accessed on 20 January 2022).

45. Elazer, B.S.S.E.; Brian, C.; Noam, J. Cardiovascular Assist System that Quantifies Heart Function and Facilitates Heart Recovery 2018. Available online: https:// patentscope.wipo.int/search/es/detail.jsf?docId=WO2018053504 (accessed on 20 January 2022).

46. Felix, J.; Dreisbach, E.M. Monitor Recorder-Implemented Method for Electrocardiography Value Encoding and Compression. 2017. Available online: https:/ / patents.google.com/patent/US9730641B2 (accessed on 20 January 2022).

47. Lim, L.S.; Wei, T.; Feng, S.G. System and Method for Assessing Clinical Event Risk Based on Heart Rate Complexity. Available online: https: / / patentscope.wipo.int/search/es/detail.jsf?docId=WO2019160504 (accessed on 20 January 2022).

48. Mark, B.N.M. Continuous Detection and Monitoring of Heart Arrhythmia Using Both Wearable Sensors and Cloud-Resident Analyses. 2019. Available online: https:/ / patentscope.wipo.int/search/es/detail.jsf?docId=WO2019108426 (accessed on 20 January 2022).

49. Wei, D. Electrocardiograph with Extended Lead Function, and Extended Lead Electrocardiogram Deriving Method. 2013. Available online: https:/ / patents.google.com/patent/US8359090B2 (accessed on 20 January 2022).

50. Raven, J.S. ECG Analysis for Diagnosis of Heart Failure and Cardiovascular Disease Using Signals Obtained from an Implantable Monitor. 2019. Available online: https:/ / patents.google.com/patent/US20190209037A1 (accessed on 20 January 2022).

51. Wu, L.; Wan, L.M.; Yang, H.-W. Multi-Chanel Real-Time Cardiovascular Performance Evaluation System and Method. Available online: https:/ / patentscope.wipo.int/search/es/detail.jsf?docId=WO2020086112 (accessed on 20 January 2022).

52. Jiang, L.; Peterson, K.T. Systems, Devices, and Methods for Non-Invasive Cardiac Monitoring. 2020. Available online: https: / / patentscope.wipo.int/search/es/detail.jsf?docId=WO2020205987 (accessed on 20 January 2022).

53. Thakur, P.H.; Ahmed, R.; Ruble, S.B. Systems and Methods for Predicting Atrial Arrhythmia. 2020. Available online: https: / / patentscope.wipo.int/search/es/detail.jsf?docId=WO2020190922 (accessed on 20 January 2022).

54. Branislav, H.L.V.; Bosko, V. Compact Mobile Three-Lead Cardiac Monitoring Device. 2020. Available online: https:/ / patentscope. wipo.int/search/es/detail.jsf?docId=WO2020232040 (accessed on 20 January 2022). 
55. Fang, D.Q. Cardiovascular Detection System and Method. 2017. Available online: https://patents.google.com/patent/US20170 065194A1 (accessed on 20 January 2022).

56. Gupta, S.; Korenberg, M. System and Method for Evaluating an Electrophysiological Signal. 2012. Available online: https: / / patents.google.com/patent/US20140194758A1 (accessed on 20 January 2022). 